EPJ Web of Conferences 31, 00041 (2012)

DOI: $10.1051 /$ epjconf/20123100041

(C) Owned by the authors, published by EDP Sciences - SIF, 2012

\title{
Silicon vertex detector for superheavy elements identification
}

\author{
A. J. Kordyasz ${ }^{1, *}$, M. KowalCZYK ${ }^{1}$, M. KISIELIŃSKI $^{2}$, \\ A. Bednarek ${ }^{1}$, M. Hadyńska-KleK ${ }^{1}$, A. Wieloch ${ }^{3}$, Z. Sosin $^{3}$, \\ D. Atanasov ${ }^{4}$, J. Sarnecki ${ }^{5}$, A. Brzozowski ${ }^{5}$, J. Jagielski ${ }^{5}$, \\ M. TeOdorczyK ${ }^{5}$, M. GAJeWski ${ }^{5}$, A. WiśniewskA ${ }^{5}$, K. KrZYŻAK $^{5}$, \\ G. GAWLiK $^{5}$, A. ZAGOJSKI ${ }^{5}$ \\ ${ }^{1}$ Heavy Ion Laboratory, Warsaw University, ul. Pasteura 5a, 02-093 \\ Warszawa, Poland \\ 2 Soltan Institute for Nuclear Studies, Świerk, Poland \\ 3 Jagiellonian University, ul. Reymonta 4, 30-059 Krakow, Poland \\ 4 University of Sofia "Sveti Kliment Ohridski", Bulgaria \\ ${ }^{5}$ Institute of Electronic Materials Technology, Warsaw, Poland
}

\begin{abstract}
Silicon vertex detector for superheavy elements (SHE) identification has been proposed. It will be constructed using very thin silicon detectors about $5 \mu \mathrm{m}$ thickness. Results of test of $7.3 \mu \mathrm{m}$ four inch silicon strip detector (SSD) with fission fragments and $\alpha$ particles emitted by ${ }^{252} \mathrm{Cf}$ source are presented
\end{abstract}

\section{Introduction}

The vertex detector very popular in high energy physics can by used for superheavy elements identification [1]. However, due to very low kinetic energy of SHE elements that are produced close to the interaction barrier, the thickness of SSD detectors should be very small about $5 \mu \mathrm{m}$. For a

${ }^{*}$ E-mail: kord@slcj.uw.edu.pl

This is an Open Access article distributed under the terms of the Creative Commons Attribution License 2.0, which permits unrestricted use, distribution, and reproduction in any medium, provided the original work is properly cited. 
such thin strip detectors the PPPP (Planar Process Partially Performed on the Thin, Silicon Membrane) process was elaborated [2,3]. The preliminary tests presented in this work with the $7.3 \mu \mathrm{m}$ thick SSD detectors made from four inch epitaxial structures, show that very thin SSD detectors can be possible to produce.

\section{Silicon vertex detector for superheavy elements identification}

The design of silicon vertex detector for identification of SHE elements is illustrated in Fig. 1. It will consist of $9 \mathrm{SSD}$ detectors of thickness about $5 \mu \mathrm{m}$ (blue color) and an additional $300 \mu \mathrm{m}$ thick detector for stopping of $\alpha$ particles emitted in forward directions (blue color) in the process of consecutive $\alpha$-radioactive decays of SHE elements. The energy loss of $5 \mathrm{MeV}$ $\alpha$ particles in $5 \mu \mathrm{m}$ thick detectors is about $0.5 \mathrm{MeV}$, which is sufficient to determine their trajectories. For $\alpha$ particles, which are emitted in backward directions, a system of $300 \mu \mathrm{m}$ thick detectors with radial strips and hole for a coming superheavy nuclei is proposed (Fig. 1, red color). Almost all $\alpha$ particles, except for those emitted parallel to the direction of SHE nuclei, are stopped by this system. The proposed vertex detector measures energies of superheavy elements as well as the products of their decay ( $\alpha$ particles and fission fragments) in a solid angle close to $4 \pi$. Additionally, it provides trajectories of all registered particles, what should considerably improves the identification of superheavy elements.

\section{Test of four inch, $7.3 \mu \mathrm{m}$ thick SSD detector with ${ }^{252} \mathrm{Cf}$ source}

For SHE elements identification by the silicon vertex detector [1] we have elaborated a new technology of large-area, thin SSD detectors. On the Fig. 2 is presented a photo of four inch silicon epitaxial $n^{+}-n$ structure with thin $7.3 \mu \mathrm{m}$ epitaxial membrane, transparent for visible light with detector strips created.

The uniformity of the thickness of the thin SSD detector was tested using ${ }^{241}$ Am source. The picture of Fig. 3 shows the $\alpha$ particle energy distribution (relative units) measured by PIN diode after crossing of the thin detector by $\alpha$ particles. Points of measurement were selected along the detector surface with $2 \mathrm{~mm}$ steps in $\mathrm{X}$ and $\mathrm{Y}$ directions. 


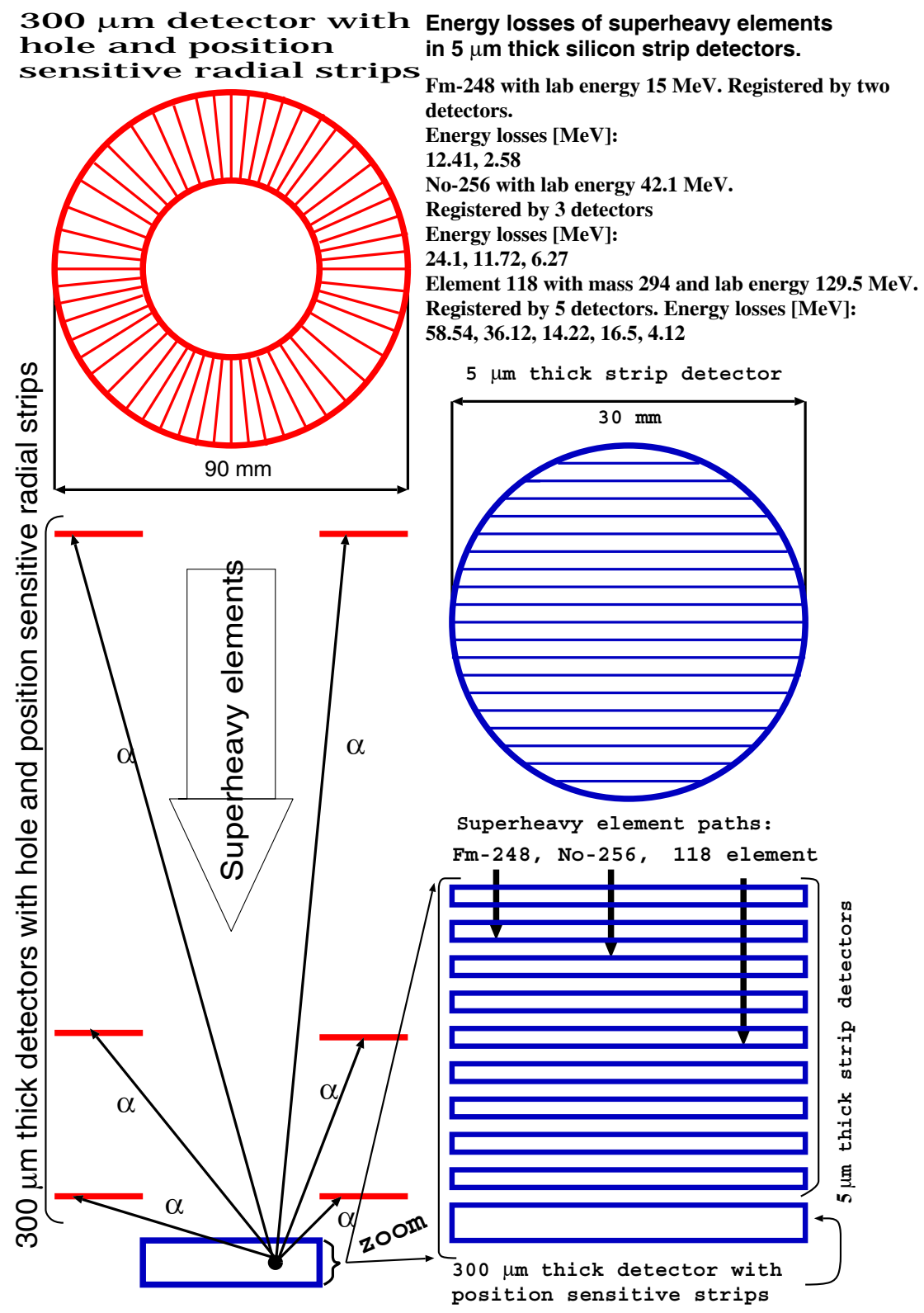

Figure 1: (Colour online) The design of silicon vertex detector for superheavy elements identification. The ranges of heavy and superheavy elements ${ }^{248} \mathrm{Fm},{ }^{254} \mathrm{No}$ and 118 element in vertex detector are presented. 


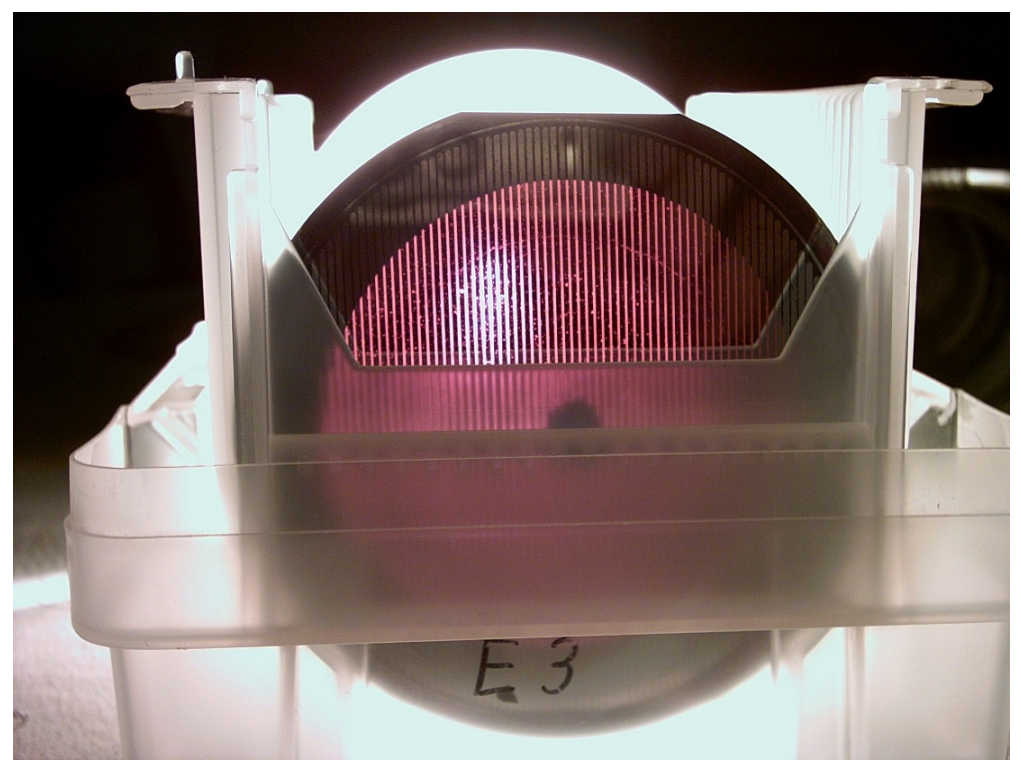

Figure 2: (Colour online) Four inch wafer with $7.3 \mu \mathrm{m}$ thin silicon membrane supported by thick $400 \mu \mathrm{m}$ silicon $\mathrm{n}^{+}$substrate ring. The detector strips are visible in the transparent light illuminating the wafer from the back side.

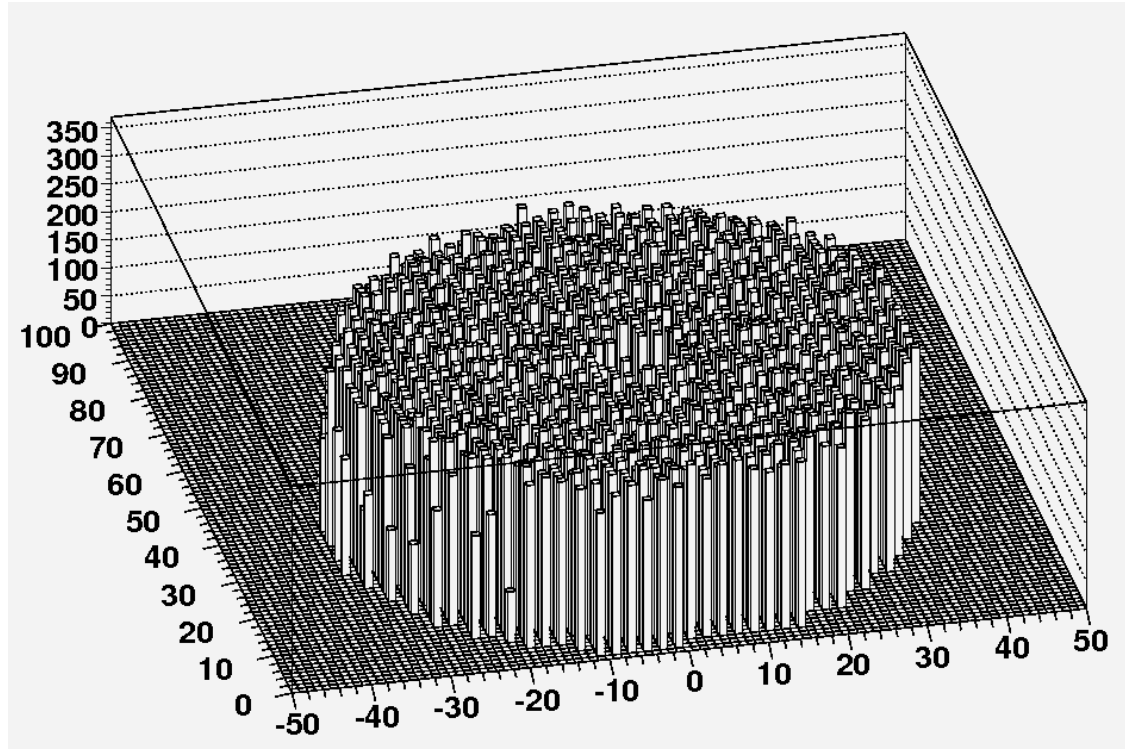

Figure 3: (Colour online) Energy lost of $\alpha$ particles from ${ }^{241} \mathrm{Am}$ in four inch wafer with $7.3 \mu \mathrm{m}$ thin silicon membrane of the strip detector. Measurements have been performed in air. 

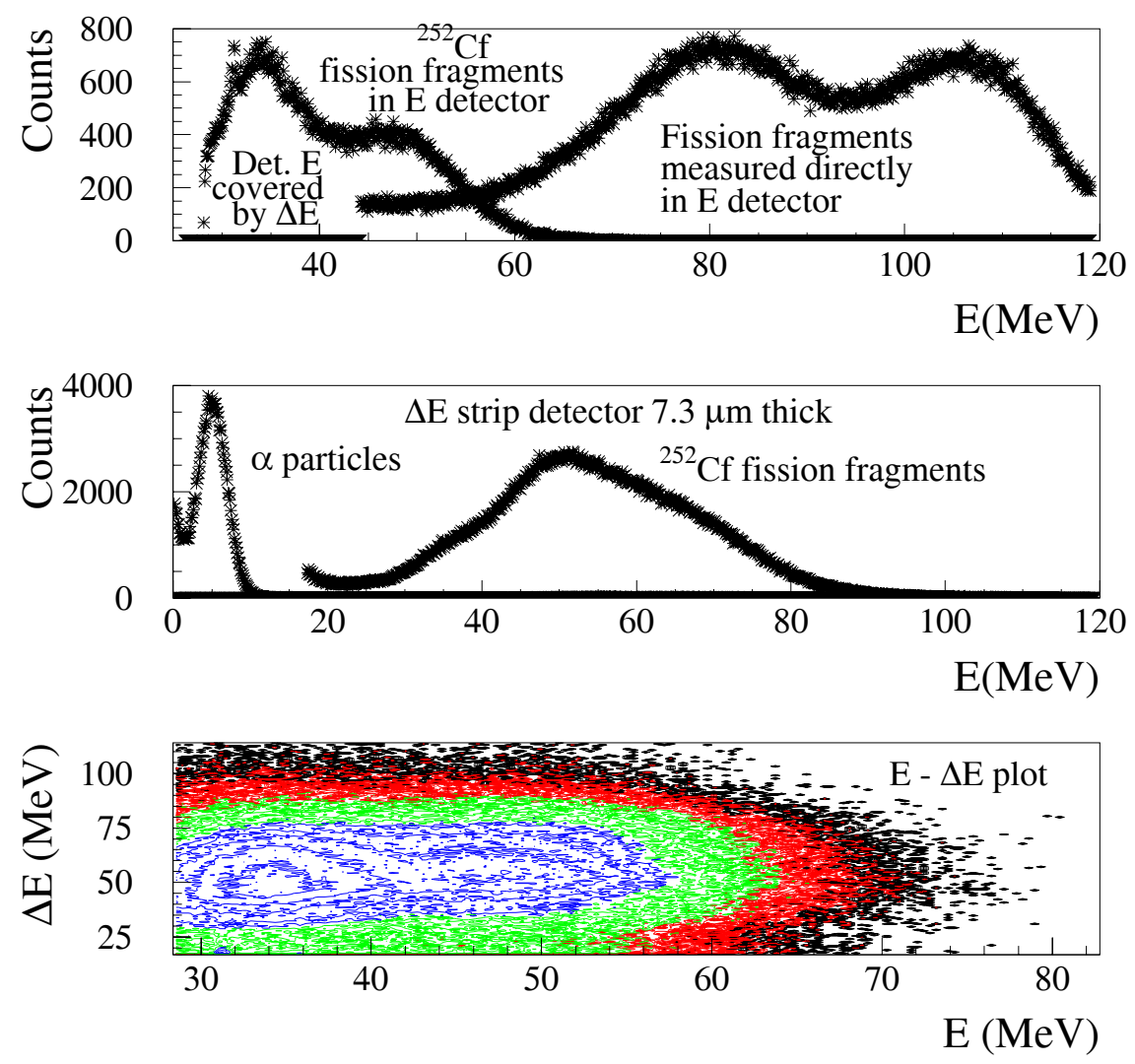

Figure 4: (Colour online) Upper figure: Fission fragments directly measured by PIN diode which is the E detector (right spectrum). After covering of PIN diode by $7.3 \mu \mathrm{m}$ SSD detector the fission fragments decreased their energy (left spectrum). Middle figure: Fission fragments (central spectrum) and $6.2 \mathrm{MeV} \alpha$ particles from ${ }^{252} \mathrm{Cf}$ (left spectrum) measured by $7.3 \mu \mathrm{m}$ strip $\Delta \mathrm{E}$ detector. The estimated energy resolution (FWHM) for $\alpha$ particles is about $0.42 \mathrm{MeV}$. Lower figure: $\Delta \mathrm{E}-\mathrm{E}$ plot measured by $\Delta \mathrm{E}-\mathrm{E}$ telescope. Left and right peaks are created by heavy and light fission fragments, respectively. 
The detector operate with very low bias potential about 1 Volt and current $780 \mathrm{nA}$. The low bias potential is probably associated with high internal, build-in field, which allow auto depletion of thin transmission detectors [2]. The $\Delta \mathrm{E}-\mathrm{E}$ telescope was mounted with collimated strip $\Delta \mathrm{E}$ detector followed by PIN diode as an E detector. The telescope was illuminated in vacuum by fission fragments and $\alpha$ particles from ${ }^{252} \mathrm{Cf}$ source. Results of measurements are presented in Fig. 4.

In conclusion, results presented in this work (Fig. 3, 4): good uniformity of the SSD and possiblity to measure energy of detected heavy ions, conviced us that one can construct very thin (e.g. $5 \mu \mathrm{m}$ ) SSD detectors and they can be very usefull in SHE detection and indentification.

\section{Acknowledgments}

We would like to thank very much to Krzysztof Rusek for fruitful discussions and comments.

\section{References}

[1] A.J. Kordyasz et al., M. Kowalczyk, A. Bednarek, J. Sarnecki, A. Brzozowski, J. Jagielski, G. Gawlik, A. Zagojski, M. Teodorczyk, G. Gajewski, Poster presented at SPIRAL2 WEEK 2011, Memorial, GANIL France

[2] A.J. Kordyasz, E. Nossarzewska-Orowska, J. Wojtkowska, M. Kisieliski, E. Kulczycka, L. Reissig, J. Kownacki, A. Wojtasiewicz, J. Sarnecki, J. Iwanicki, Nucl. Instr. and Meth. A 539 (2005) 262

[3] A.J. Kordyasz, E. Nossarzewska-Orowska, J. Wojtkowska, M. Kisieliski, E. Kulczycka, L. Reissig, J. Kownacki, A. Wojtasiewicz, J. Sarnecki, J. Iwanicki, Nucl. Instr. and Meth. A 570 (2005) 336 